\title{
Instrument for Lunar Seismic Activity Studies on Chandrayaan-2 Lander
}

\author{
J. John*, V. Thamarai, M. M. Mehra, Teena Choudhary, M. S. Giridhar, \\ Ashwini Jambhalikar, Gogulapati Supriya, Gaurav Saxena, K. V. Shila, \\ B. Ramesh, T. K. Pratheek, Deepak Kumar Sharma, R. Islam, P. Selvaraj, \\ A. Kalpana, S. Ajith Kumar, K. V. Sriram and A. S. Laxmiprasad \\ Laboratory for Electro-Optics Systems, Indian Space Research Organisation, Peenya 1st Stage, 1st Cross, Bengaluru 560 058, India
}

Instrument for Lunar Seismic Activity Studies (ILSA) is a science payload with the objective of studying seismic activities at the landing site of Vikram, the Lander of Chandrayaan-2. ILSA will be deployed to the lunar surface by a specially built mechanism. It is an indigenously developed instrument based on microelectro mechanical systems technology. High sensitivity silicon micro-machined accelerometer is the heart of the instrument that measures ground acceleration due to lunar quakes. The instrument has the capability of resolving acceleration better than 100 nano-g $\mathrm{Hz}^{-1 / 2}$ up to a range of $0.5 \mathrm{~g}$ over bandwidth of $40 \mathrm{~Hz}$. This paper presents the basic concepts in the design, realization, characterization and the performance test results of the space qualified strong motion seismic sensors.

Keywords: Lunar quakes, MEMS, seismometer, strong motion sensors.

SEISMOLOGY is the best geophysical tool to determine the internal structure of a planet. Seismometers with varying range of performance parameters both in terms of sensitivity and bandwidth are used to conduct seismic experiments. The most detailed study of lunar seismology was conducted during the Apollo programme by the US during 1964-1977. The experiments conducted by Apollo $11,12,14,15$ and 16 missions have recorded about 12,000 seismic events classified into various types ${ }^{1,2}$. The analysis of these events provided tectonics of the Moon, Moonquake characteristics, state of stress and ground acceleration models as reported by Watters et al. ${ }^{3}$. There are still several unanswered questions in lunar seismology and several space agencies, with a renewed interest, are putting efforts to continue the studies. With advanced technologies, instrumentation and data processing capabilities, new facts can be obtained in lunar seismology. Pertaining to Mars seismology, NASA's Mars Insight lander with its Seismic Experiment for Interior Structure (SEIS) has recorded faint seismic signal on 6 April 2018

\footnotetext{
*For correspondence. (e-mail: jjohn@leos.gov.in)
}

and is considered to be the first recorded 'mars quake'. The SEIS instrument consists of six axes seismometer and is expected to obtain much improved knowledge of the interior structure of Mars ${ }^{4}$. The only attempt to study the seismology of Venus was by the Soviet missions Venera 13 and 14 during 1982. The highly hostile environment of Venus made the mission too short lived to obtain meaningful data ${ }^{5}$. Active research and development is going on to overcome the problems by developing new technologies and advanced instrumentation methods ${ }^{6}$.

Instrument for Lunar Seismic Activity Studies (ILSA) will be the first instrument of its type to be placed at the identified landing site in the south pole of the Moon. The instrument has three axes high sensitivity accelerometers realized using the silicon micromachining technology called micro-electro mechanical systems (MEMS). ILSA has the objective of characterizing the seismicity around the landing site. The data obtained from ILSA will be first classified and catalogued by the instrument development team at the author's laboratory. Subsequently, the detailed analysis will be conducted by a group of scientists led by National Geophysical Research Institute, Hyderabad.

\section{ILSA configuration}

The amplitude and frequency ranges of seismic signals in the form of ground acceleration are very wide making the instrument design extremely challenging. The signal amplitude levels of earthquakes are in the range of nano$\mathrm{g}$ to $\mathrm{g}\left(\mathrm{g}=9.8 \mathrm{~ms}^{-2}\right)$ and the frequency band is from 0.0001 to several tens of Hertz ${ }^{7}$. This could be the basic design guideline for instruments to measure lunar quakes as well. Generally a single instrument cannot cover the entire range of interest. ILSA falls under the class of broadband strong motion sensors with dynamic range designed to be better than 100 nano-g $\mathrm{Hz}^{-1 / 2}$ to $0.5 \mathrm{~g}$ over a bandwidth of $40 \mathrm{~Hz}$. The overall specifications of the instrument are summarized in Table 1. ILSA has its sensors fabricated using silicon MEMS technology offering multiple inherent advantages of being of less payload 
mass and having low power consumption. ILSA is a cluster of accelerometers distributed over three orthogonal directions. There are two of them along an axis and are designated as coarse range and fine range sensors and are capacitive transducers. The output from the coarse range sensor is processed by capacitance to digital converter IC and that from the fine range sensor is processed by discrete capacitance to voltage converter circuit. The overall instrument architecture is represented in the block diagram given in Figure 1.

The readout electronics employs an open loop sensing scheme. The fine range sensors are designed to operate up to signal amplitude of 1.5 milli-g whereas the coarse range sensor has range until $0.5 \mathrm{~g}$. There is an overlapping range of operation for both of them. The sensors and the front end electronics are housed in hybrid micro circuit (HMC) package. The power supply regulation, FPGA circuitry for generation of various clocks and digitizing of signals are all implemented in printed circuit boards. All these modules are assembled in a single mechanical housing with a provision to deploy it to the lunar surface from its location of mounting in the Lander. The photograph of the instrument is shown in Figure 2.

The basic building blocks of the instrument are explained in detail in the following sections.

\section{Micro-electro mechanical systems sensing elements}

The sensing element consists of beam-plate system micro-machined from single crystal silicon wafer using standard processes in MEMS technology. It has silicon on glass architecture where the microstructure is supported on a glass wafer. The heart structure is a proof mass suspended by four-folded beams and have comb electrodes defined on it. These combs are inter-digitated with fixed comb electrodes to form a capacitor. The comb electrodes are distributed as four sets at both sides of the proof mass

Table 1. Overall specifications of instrument for lunar seismic activity

\begin{tabular}{ll}
\hline Specification & \multicolumn{1}{c}{ Value } \\
\hline Instrument type & $\begin{array}{c}\text { Three axis, micro-electro mechanical } \\
\text { systems (MEMS)-based }\end{array}$ \\
& \pm 0.5 \\
Dynamic range $(\mathrm{g})$ & 100 \\
Resolution $\left(\right.$ nano-g/ $\left./ \mathrm{Hz}^{1 / 2}\right)$ & 40 \\
Bandwidth $(\mathrm{Hz})$ & -20 to +45 \\
Operating temperature $\left({ }^{\circ} \mathrm{C}\right)$ & -50 to +85 \\
Storage temperature $\left({ }^{\circ} \mathrm{C}\right)$ & 1.8 \\
Weight $(\mathrm{kg})$ & $170 \times 170 \times 72$ \\
Size $(\mathrm{mm})$ & $<4$ \\
Power consumption $(\mathrm{W})$ & $\pm 15 \mathrm{~V},+6 \mathrm{~V}$ \\
Power supply (voltage) & Serial digital \\
Output & $<1 \%$ \\
Cross axis sensitivity & Hold down and release mechanism \\
Deployment & through Frangibolt assembly \\
&
\end{tabular}

to form a differential capacitor assembly. This arrangement helps to minimize cross axis sensitivity of the instrument. By virtue of the design of the beams, the sensor has its sensitive axis in one specified direction of its plane and is stiffer along all other directions. Stoppers are provided to protect the structure from having large deflection due to mechanical shocks. The schematic representation of the sensor is shown in Figure 3.

\section{Working principle}

The working principle of the capacitive sensing accelerometer in ILSA is schematically represented in Figure 4. The MEMS structure represented in Figure 3 constitutes single degree of freedom spring-mass-damper system and obeys the classical forced harmonic motion principles when acted upon by external acceleration ${ }^{8}$.

$$
m \frac{\partial^{2} x}{\partial t^{2}}+c \frac{\partial x}{\partial t}+k x=m \frac{\partial^{2} u}{\partial t^{2}},
$$

where $m$ is the proof mass, $x$ its deflection, $c$ the damping constant, $k$ the stiffness of the cantilever suspension structure and $u$ is the ground displacement. Mechanical sensitivity is defined as the deflection of the proof mass $x$ per unit $g$ of acceleration. The mass of the structure and its mechanical stiffness determines the mechanical sensitivity as given by the classical spring equation given below, which is also the solution to the eq. (1) approximated for static and quasi static range of input.

$$
F_{e}=m a=k x .
$$

The sensor is arranged to form a capacitor assembly where one of the electrodes is a part of the spring-mass system, whereas the other electrode is fixed relative to the former with an initial separation of $d_{0}$. By design, the separation between electrodes changes as a function of external input acceleration. The capacitance value associated with the assembly, defined as dead value, $C_{0}$ is given by

$$
C_{0}=\left(\frac{\varepsilon_{0} A}{d_{0}}\right),
$$

$\varepsilon_{0}$ is the permittivity of free space and $A$ is the area of overlap between electrodes.

When there is external acceleration and deflection of movable electrode, the associated capacitance changes to $C^{\prime}{ }_{ \pm}$as

$$
C_{ \pm}^{\prime}=\left(\frac{\varepsilon_{0} A}{d_{0} \mp x}\right) .
$$

For small deflections, the change in output $\Delta C$ is proportional to the magnitude of deflection of the proof mass 


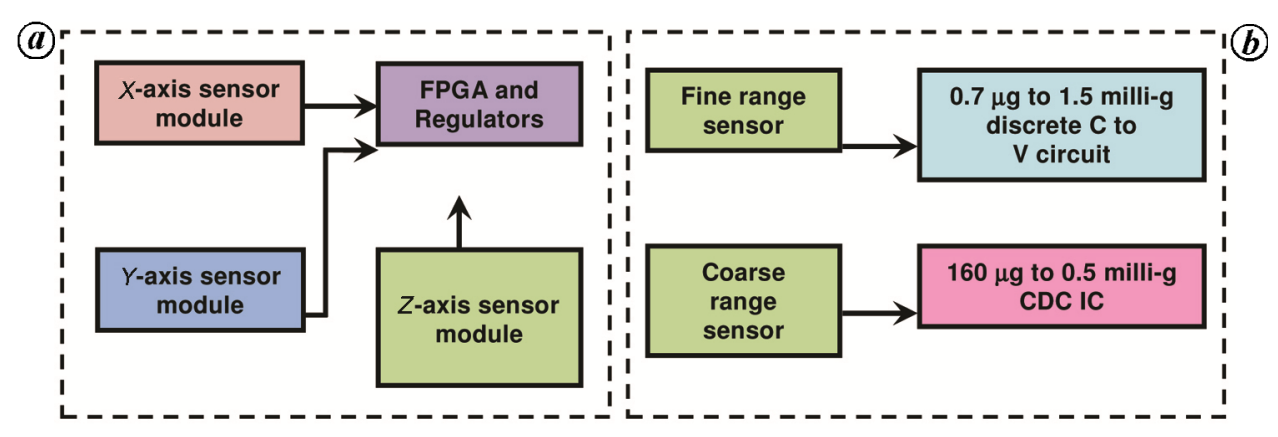

Figure 1. $\boldsymbol{a}$, Overall architecture of the instrument. $\boldsymbol{b}$, Configuration of sensor module along each axis.

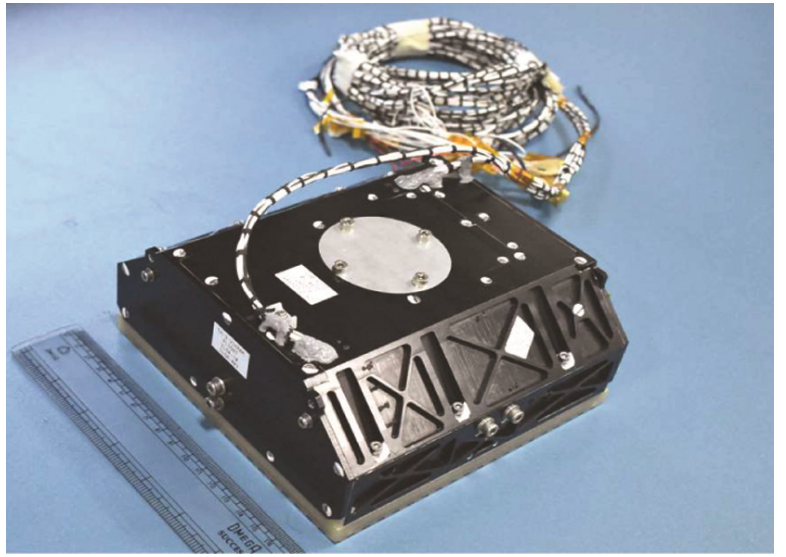

Figure 2. Photograph of instrument for lunar seismic activity (ILSA) package.

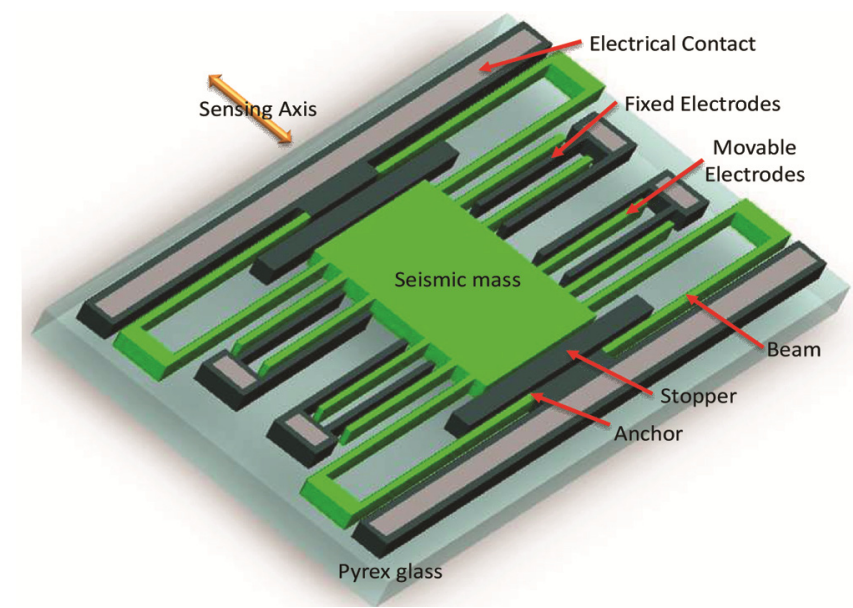

Figure 3. Schematic representation of silicon microstructure acting as the sensing element.

which in turn is a function of input acceleration. In open loop accelerometers with large deflection of proof mass, the output will be highly non-linear ${ }^{9}$. The fine range sensor of ILSA has a deflection of less than $1 \%$ of $d_{0}$ to ensure linearity in output. The output change in differential mode is picked up and processed by suitable readout electronic circuit.

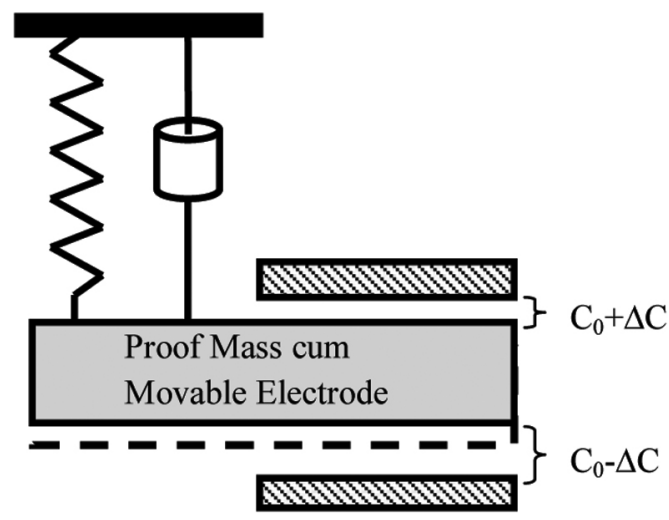

Figure 4. Schematic representation of working principle of ILSA sensing element.

The main difference between fine range and coarse range sensors is in the size of the proof mass which affects the Brownian noise equivalent acceleration that decides the resolution of the instrument ${ }^{10}$.

\section{Sensor fabrication}

The sensor is fabricated from low resistivity silicon wafer by bulk etching process. The key process steps of fabrication are: (i) the Deep Reactive Ion Etching of silicon by Bosch process to define the high aspect ratio features; (ii) anodic bonding to join glass and silicon wafers; (iii) contact definition by aluminium patterning and wet chemical etching; (iv) trench dicing, and (v) release etching in addition to regular photolithography processes prior to all etching steps. Figure 5 shows the photograph of the sensors and the SEM image of a portion of the sensor showing the electrode assembly. The details of fabrication are reported in ref. 11 .

\section{Readout electronics}

The coarse range sensor has a capacitance to digital converter IC from analog devices and has serial output with $\mathrm{I} 2 \mathrm{C}$ interface. The fine range sensor has an op-amp based 


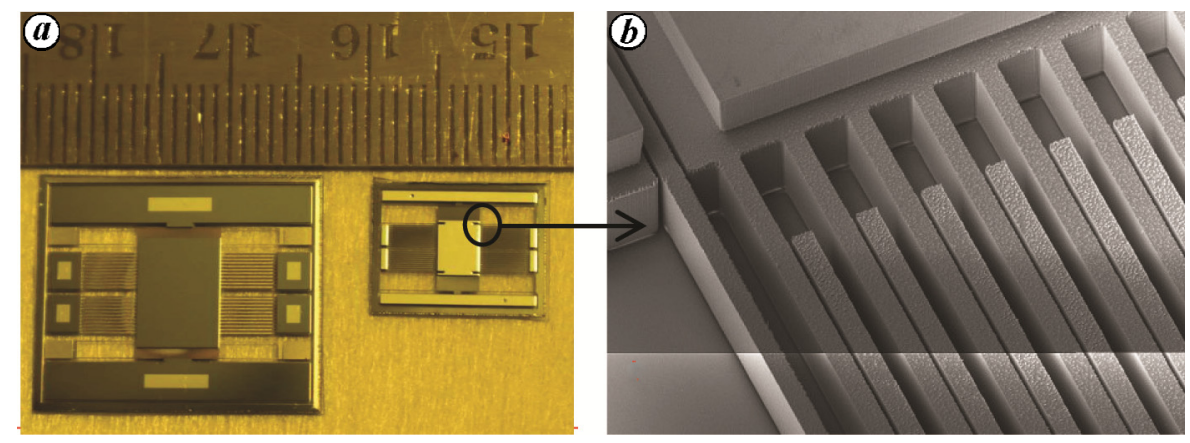

Figure 5. Photographs of coarse and fine range MEMS sensor elements (a) with SEM image showing the electrodes $(\boldsymbol{b})$

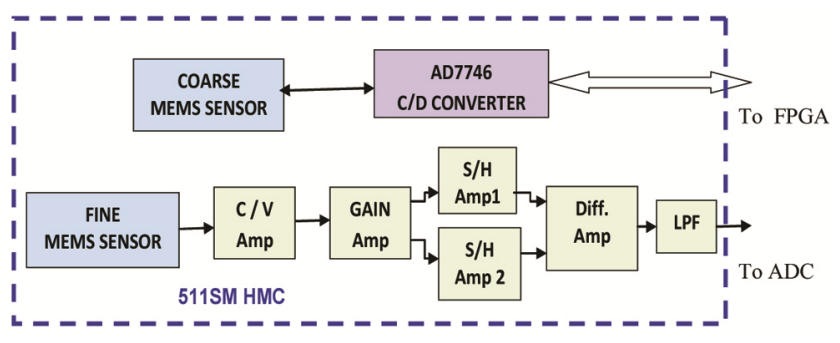

Figure 6. Block diagram of front end electronics of ILSA.

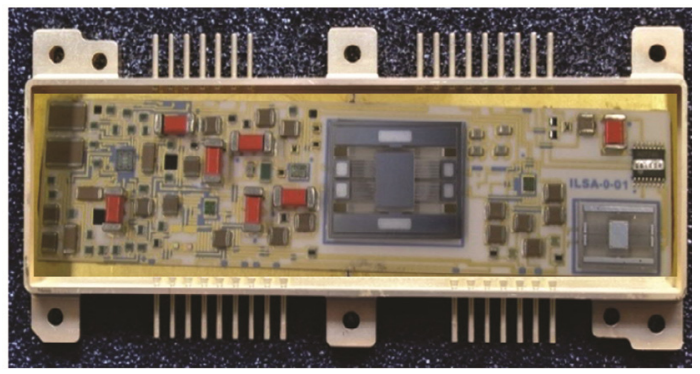

Figure 7. Photograph of $1^{\prime \prime} \times 4^{\prime \prime}$ hybrid micro circuit with sensors and front end electronics for ILSA.

capacitance to voltage conversion circuit, amplifying and filtering electronics and is realized with discrete components. The block diagram of front end readout electronics is shown in Figure 6.

This is realized as a custom designed HMC package whose photograph is shown in Figure 7.

The HMC package is hermetically sealed in dry nitrogen ambient at 1 atmosphere pressure.

The generation of clock signals for excitation of MEMS sensor, SDA and SCLK for I2C interface, analog to digital conversion of signal from fine range sensor, formatting of data to communicate to command telemetry data storage package are all done through RTSX72SU FPGA. The instrument delivers 120 bits data every 5 milliseconds containing output from all six sensors along with other health status information including the temperature of the HMCs. This data with a volume of $24 \mathrm{kbps}$ is time-stamped and stored in solid state recorder and will be periodically sent to the data receiving centre.

Conventional seismometers are aligned and placed manually ensuring their orientation on a levelled platform. But in the present case manual deployment is not possible. There is a chance that due to undulations on lunar regolith, the sensitive axis of the instrument will not be aligned as expected with respect to the surface normal. Being an accelerometer operating in open loop mode, the gravity component will act as input, shifting the output offset of the instrument. Since the acceleration due to gravity at Moon is less and the coarse range sensor has a wide operating range, it will not be saturated even at highly tilted placement. The time varying signals due to ground accelerations could be recorded over the offset value without loss of information. But since the fine range sensor has a limited upper range of operation, there is a need of implementing tilt corrections after the placement of instrument on lunar surface. A unique way of electronic tilt correction is implemented for the fine range sensor in order to achieve this objective. Here the instrument is brought to operation range in case of its tilted placement by providing corrective voltages to the amplifier inputs by ground commands. The tilt information for correction is obtained from the offset of coarse range sensor. This approach helps to overcome the limitations in operating range imposed by the open loop readout employed in the instrument. This corrective mechanism can also be used to compensate for the offset drift of the fine range sensor with temperature over the entire range mentioned in Table 1.

The instrument has another module that regulates the power supply received from the DC-DC converter. The power cards, FPGA card, HMCs are all integrated in a mechanical housing along with the electrical and data harness wires. The instrument communicates to the central computing and data processing unit of the Lander to send and receive data. The package is provided with electrical heaters to ensure its operation within the specified temperature limits. The base of the ILSA package is a thick glass fibre reinforced plastic (GFRP) for thermal isolation from extremely cold lunar soil. The thermal 

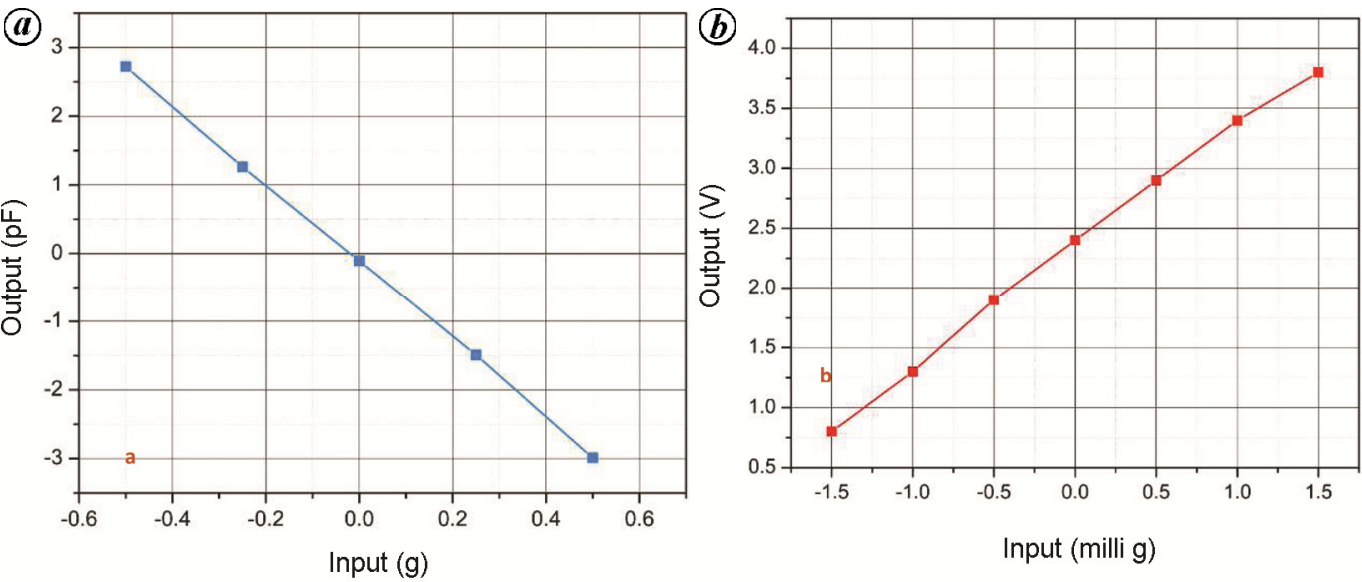

Figure 8. Response of coarse range (a) and fine range (b) sensors.

design of the package has been arrived at after detailed modelling and analysis considering the landing site and ambient thermal conditions both in illuminated and shadowed conditions. The GFRP base is provided with grooves to ensure good contact of the instrument with the lunar regolith. Special mechanisms are not employed to ensure very strong coupling of instrument to ground. Since seismic activities generate low frequency low amplitude ground vibrations, the coupling resulting from weight of the instrument is sufficient to detect the signals.

\section{ILSA calibration}

Being high sensitive accelerometer, ILSA adopts calibration standards for linear accelerometers ${ }^{12}$. High precision rotation table that is levelled and calibrated for its performance specifications is used to give a known rotation to impart a known gravitational component input to the instrument. The accuracy of calibration also depends on the knowledge of $g$ value at the location of making the measurements. This value was provided to the authors to a precision of one micro-g by the field testing team of Survey of India. The output of the instrument is recorded against the input corresponding to its dynamic range. This helps to obtain the offset, scale factor, linearity and cross axis sensitivity of the instrument. The tests are repeated at various temperatures to estimate the thermal drift of these parameters. The noise equivalent acceleration or the resolution of the instrument is measured by recording output over a period of time and estimating peak to peak magnitude. This has to be done at a location where the ambient cultural noise is less than the electronic noise of the instrument and it is found to be difficult to identify a place meeting that criterion. The output from ILSA was recorded at various locations and at various points of time over the day to characterize the noise performance. The minimum values obtained so far by experiment are claimed to be the resolving capability of the instrument.

\section{Test results}

This section summarizes the test results obtained from the $X$-axis sensor of the qualification model of the instrument which represents the typical response from other sensors as well.

The coarse range sensor is calibrated over an input of $\pm 0.5 \mathrm{~g}$ and the response is given in $\mathrm{pF} / \mathrm{g}$. The fine range sensor is calibrated over \pm 1.5 milli-g and the response is expressed as $\mathrm{V} / \mathrm{g}$. Figure 8 shows the response of the sensors.

The figure shows that the coarse range sensor has sensitivity around $5.7 \mathrm{pF} / \mathrm{g}$ and fine range sensor has around $1014 \mathrm{~V} / \mathrm{g}$.

The performance of ILSA has been tested from -20 to $+45^{\circ} \mathrm{C}$ temperature range. It was found that the scale factor drift with temperature is negligible. However, there is a temperature dependent offset drift which was effectively corrected with the mechanism described in the previous section.

From the recording obtained from ILSA from various locations over different points of time it is found that peak to peak output recorded during early morning hours from a specific location with well-built vibration isolation platform showed the minimum practically obtained value of around 100 nano-g $\mathrm{Hz}^{-1 / 2}$. Figure 9 shows the output from ILSA representing the resolution capability of the instrument. Here the output from the instrument as shown in the $y$-axis is around $1 \mu \mathrm{g}$ peak to peak. The resolution is expressed by dividing this magnitude by the square root of bandwidth of the instrument.

The response of the instrument to dynamic input has been characterized by using shaker tables. The hydraulic shaker has the capability to provide low frequency, low amplitude mechanical input. However, there are some limitations in conducting the test over the entire range of interest due to machine limitations and the noisy environment as the shaker is initiated for test.

The bandwidth of the fine range sensor is estimated separately by analysing its response to an input given by 


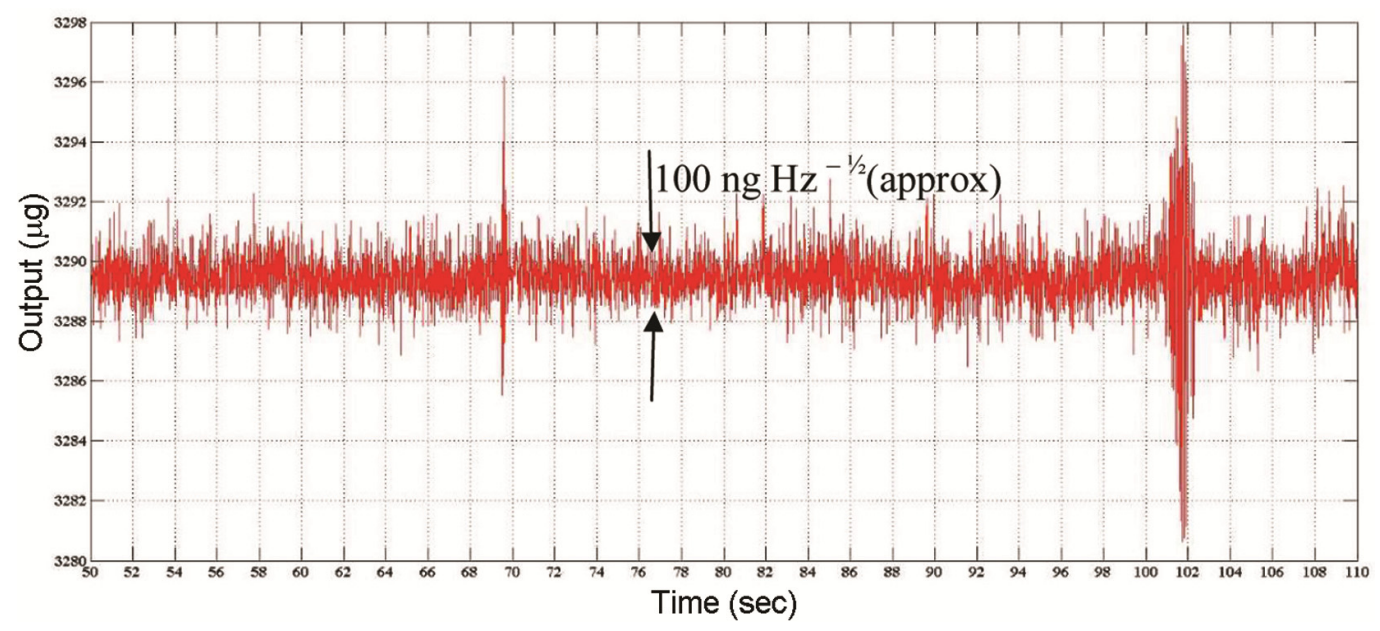

Figure 9. Resolution capability of ILSA with $1 \mu \mathrm{g}$ peak to peak noise calculated to resolution of 100 nano-g Hz $\mathrm{Hz}^{-1 / 2}$.

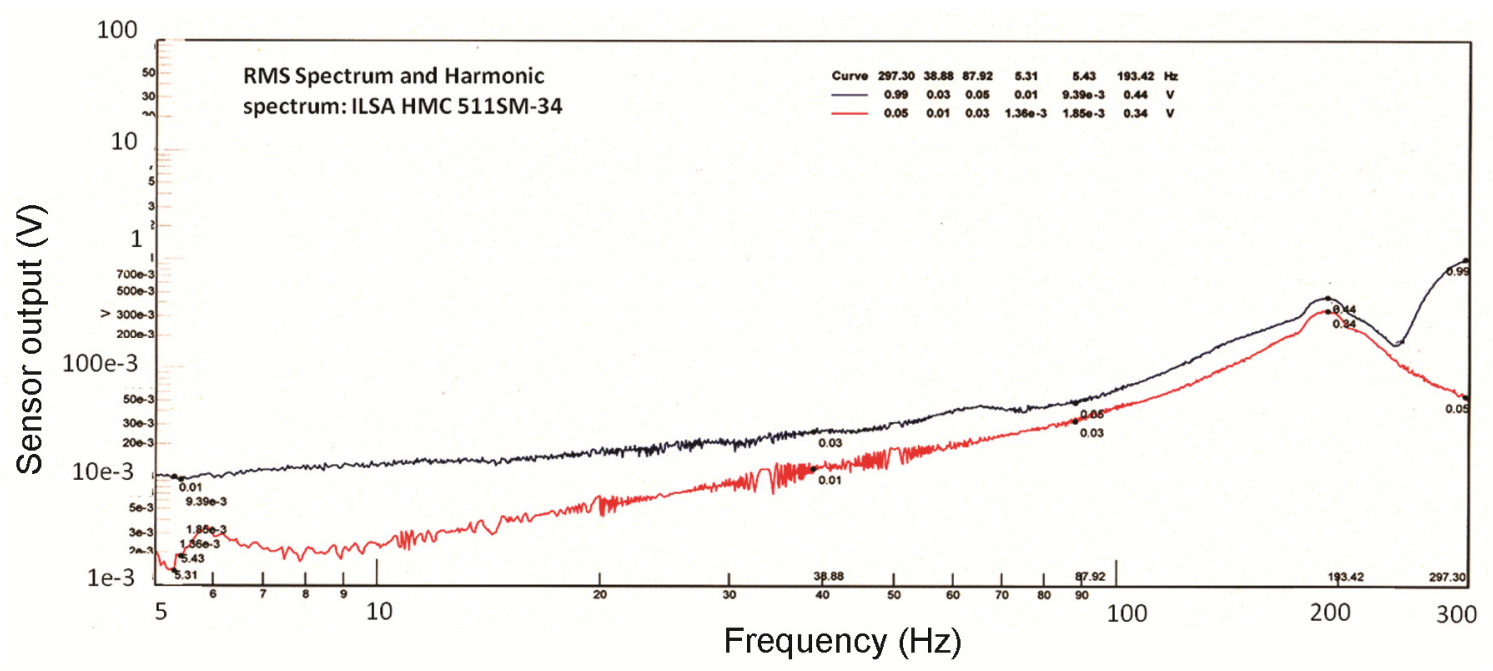

Figure 10. Dynamic response of fine range sensor showing flat bandwith of operation.

an electrodynamic shaker. In the test, the output from the sensor as an analog voltage from an intermediate stage has been recorded as the input of $0.5 \mathrm{~g}$ is swept over frequency range till $300 \mathrm{~Hz}$. Figure 10 shows the response of the fine range sensor. The $y$-axis shows the output voltage from the fine range sensor before its amplification and the $x$-axis shows the frequency range of sweep. The data shows that the sensor has a flat response over the entire bandwidth of interest for the seismic signals $(40 \mathrm{~Hz})$ and the sensor has resonance peak at around $200 \mathrm{~Hz}$.

In order to compare the performance of ILSA with a commercially available seismometer from Trillium, both the instruments have been simultaneously powered on at a given location. Both showed same amplitude of signals of the order of several $\mu \mathrm{g}$ peak to peak indicating the cultural noise at the test location changing at various hours of the day.
ILSA underwent the qualification tests successfully to ensure the reliability of the instrument in the operating environment. The tests include thermo-vacuum tests, hot and cold storage, sine and random vibrations and mechanical shock tests as demanded by the mission.

\section{Conclusion}

Instrument for lunar seismic activity studies on Moon is an indigenously developed MEMS-based instrument. It is first of its kind to function as strong motion earthquake sensors. ILSA is meeting its targeted specifications giving the hope that it can extract meaningful seismic signals from the landing site of Vikram of Chandrayaan2. Fine tuning the design and performance parameters of ILSA can result in very useful spin off product - that is, indigenous, compact seismometers for Earth-based 


\section{RESEARCH ARTICLES}

applications. Being a MEMS-based instrument, batch production of sensors can be achieved enabling the possibility of realizing seismic sensor network for structural health monitoring for a variety of applications.

1. Bulow, R. C. et al., New events discovered in the Apollo lunar seismic data. J. Geophys. Res., 2005, 110, E10003.

2. Yamada, R., The description of Apollo seismic experiments; www.darts.isas.jaxa.jp/planet.seismology.

3. Watters, T. R. et al., Shallow seismic activity and young thrust faults on the Moon. Nature Geosci., 2019, 12, 411-417; https://www.nature.com/articles/s41561-019-0362-2.pdf

4. Lognonne, P. et al., SEIS: insight's seismic experiment for internal structure of mars. Space Sci. Rev., 2019, 215, 12; https:// doi.org/10.1007/s11214-018-0574-6

5. Measuring seismic activity on Venus: a real challenge; https://www.seis-insight.eu/en/public-2/planetary-seismology/venus

6. Hunter, G. et al., Development of a high temperature Venus seismometer and extreme environment testing chamber. International Workshop on Instrumentation for Planetary Missions, 2012.

7. Havskov, J. and Alguacil, G., Instrumentation in Earthquake Seismology, Springer, June 2002; doi:10.1007/978-3-319-21314-9, ISBN 1402029683.

8. Kumar, S., Design and Fabrication of Micromcahined Silicon Suspensions, Ph D thesis, Imperial College, London, 2007.

9. Kempe, V., Inertial MEMS Principles and Practice, Cambridge University Press.

10. Kulah, H. et al., Noise analysis and characterization of a sigmadelta capacitive microaccelerometer. IEEE J. Solid-State Circuits, 2006, 41(2), 352-361.

11. John, J. et al., Design and fabrication of silicon micro structure for seismometer, ISSS International Conference on Smart Materials, Structures and Systems, Bengaluru, 2014.
12. IEEE standard specification format guide and test procedure for linear single axis nongyroscopic accelerometers IEEE Std 1293 , 1998 (R2008).

ACKNOWLEDGEMENTS. ILSA team would like to express their heartfelt thanks to all those people and institutions who supported the development and testing of this product. We thank Dr T. K. Alex, Shri. J. A. Kamalakar and Dr G. N. Rao, former Directors of LEOS and Shri M. Vishwanathan, former Group Director of LEOS, for the support and encouragement extended at every stage of development of this instrument. We are thankful to R. P. Selvam and team at Earthquake Engineering and Vibration Research Centre, Central Power Research Institute (CPRI), Bengaluru for extending the test facilities. We acknowledge the support from Seismology Division of Bhabha Atomic Research Centre (BARC), Gauribidinur for the technical support extended by the team at the initial stages of the development of the instrument. The invaluable support from National Geophysics Research Institute, Hyderabad and various teams of U.R. Rao Satellite Centre (URSC) are thankfully acknowledged. We specially thank the mechanisms and thermal teams of URSC for developing the hardware for the deployment of ILSA and conducing detailed thermal calculations. We are also thankful to Karnataka Hybrid Micro Devices Limited (KHMD), Bengaluru for fabricating, screening and qualifying HMCs required for ILSA. We express our gratitude to Sensor Assembly Fabrication Facility of LEOS for neatly integrating the instrument and Environment Test Facility Team for conducting a series of vibration and thermovacuum tests to ensure its reliability.

Received and accepted 27 August 2019

doi: $10.18520 /$ cs/v118/i3/376-382 